\title{
Estimating shoulder-complex mobility
}

doi:10.1080/11762320701403922

\section{Bertrand Tondu}

LESIA, Département de Génie Electrique, Institut National de Sciences Appliquées, Campus Universitaire de Rangueil, 31077 Toulouse France

\begin{abstract}
Modeling shoulder motions is essential in understanding upper-limb dynamic behavior. However, the mobility of the shoulder complex — in terms of degrees of freedom (DOFs) — is seldom quantified. In this paper we propose applying the theory of mechanisms and, especially, the powerful Grübler-Kutzbach formula to estimate it. This approach requires a rigorous kinematic analysis. So we suggest basing it on a bone schematisation derived from an extension of Dempster's historical notation associated to mechanical interpretation of the physiological joints involved. In so doing, the proposed shoulder complex mobility leads to justifying a nine-DOF serial-chain upper-limb mode, which extends the classical seven-DOF arm-foream-wrist model and completing it by adding a clavicle-like link with a two-DOF mobility.
\end{abstract}

Key words: Shoulder complex, mobility, kinematic modelling of bones and joints.

\section{INTRODUCTION}

The shoulder complex is often presented as the most mobile kinematic system of the human body. Curiously this mobility is seldom quantified. Two reasons seem to be related to this lack. First, the shoulder complex is not moved by a single joint but by a complex set of joints, including the shoulder girdle closed chain and its specific 'false scapulothoracic joint' (Kapandji 1982). ${ }^{1}$ Second, this kinematic complexity is associated to a complexity of the musculature that actuates the shoulder joints. Even today certain authors still highlight this difficulty in understanding the kinematic properties of the shoulder joint complex: 'However, the shoulder joint complex is an articulation that defies simple kinematic description' (Rab et al. 2002, p 113), or in this near admission of ignorance: 'The shoulder joint is a complex joint with many degrees of freedom' (Magermans et al. 2005, p 591). A consequence of the difficulty in determining the mobility of the shoulder complex

\author{
Corresponding Author: \\ Bertrand Tondu \\ LESIA, Département de Génie Electrique \\ Institut National de Sciences Appliquées \\ Campus Universitaire de Rangueil, 31077 Toulouse, France \\ Tel: (33) 5622667 42; Fax: (33) 561559800 \\ E-mail: bertrand-tondu@insa-toulouse.fr
}

\footnotetext{
${ }^{1}$ Applied to joint physiology, the term false is generally used to specify a joint that is not rigorously speaking an anatomical joint of a given type, but results from the sliding of two surfaces with respect to each other, maintained in contact by surrounding tissues. In the case of the 'false scapulothoracic joint', which plays a very important role in shoulder-complex mobility, this is the surrounding musculature that maintains the scapula in contact with the thorax.
}

leads to giving the scapula a number of degrees of freedom (DOF) depending highly on the study framework. Mentioning only recent studies, the scapula has nil mobility in Scepi et al. (2004) and a mobility of five DOFs in the Comprehensive Human Animation Resource Model (CHARM Esprit Project; Maurel and Thalmann 1999, 2000), that is, practically the two extreme values allowed for by mechanics for a single link. Several recent studies simply avoid the question of shoulder complex mobility estimates either by kinematically defining the scapula as an independent solidly oriented entity with respect to the thorax due to three Euler angles (Karduna et al. 2000; McClure et al. 2001; Dayanidhi et al. 2005), or by considering an empirical model of the scapula, as given in the recent Stanford University model (Holzbaur et al. 2005), which animates the shoulder complex and its musculature by using De Groot's regression equation (De Groot 2001).

In the framework of this paper, we would like to show that the application of the theory of mechanisms to the physiology of joints can help in the understanding of shoulder-complex kinematics, notably in determining shoulder girdle mobility using the Grübler-Kutzbach formula. Our article is divided into four sections. In the first section, we deal with the diagrammatic presentation of jointed body links. An extension of Demspter's historical notation seemed to us to be indispensable in clarifying the kinematic diagrams and extending them to multiple joints. By associating this link-notation with a symbolic representation of the joints in their physiology, inspired from mechanical joint symbols, a general notation for a conventional representation of kinematic chains of the skeletal system is proposed. In the second section, having first justified the move from the joint physiology point of view of our choices 
Table 1 General correspondence between joint physiology and classical mechanism theory

(1)

for the kinematic nature of the main shoulder joints, we put forward a conventional kinematic representation of the shoulder complex. In the third section, an evaluation of the mobility of the shoulder complex based on our kinematic analysis of the shoulder girdle is given. In the fourth, we consider the possibility of defining an open-chain kinematic model equivalent to the human shoulder complex, leading us to validate a nine-DOF kinematic model of the human upper extremity, which can appear as an extension of the usual seven-DOF model of the upper limb considered in biomechanism (Raikova 1992; Biryukova et al. 2000; Prokopenko et al. 2001; Abdel-Malek et al. 2004) by adding a clavicle-like link to it.

\section{KINEMATIC REPRESENTATION OF JOINTED BODY LINKS}

Joint Physiology makes a distinction between three kinds of joints: diarthroses (also known as synovial joints), which are mobile; amphyarthroses, which are semimobile; and synarthroses, which are immobile. Only diarthroses are really concerned in the analysis of human motion. They are generally classified according to the geometric shapes of their joint surfaces, into the following categories:

- ball-and-socket joints, with spherical-like surfaces, giving three rotation motion possibilities to the joint;

- flat joints, whose surfaces are flat, i.e., less curved so as to be considered ideally as a flat surface; generally speaking, the flat joint allows two translation motions on a plane and one rotation motion perpendicular to this plane;

- condyl or ellipsoidal joints, whose surfaces are ellipsoidal, allowing flexion-extension and abductionadduction motions, but excluding the ball-and-socket spinning motion;
- saddle-shaped joints, the surfaces of which are reciprocally concave-convex, allowing flexion-extension and abduction-adduction motions as in condyl joints;

- hinge joints, whose cylindrical-like surfaces are apposed, generating a pulley-like rotation motion;

- trochoid or pivot joints, where the surfaces are also cylindrical-like so as to generate a rotation motion around a pivot.

Owing to the specificity of articular joint surfaces, this taxonomy of physiological joints is original compared to the standard classification of mechanical joints. It is subsequently possible to suggest an equivalence between physiological and fundamental joints as given in Table 1.

Such a mechanical interpretation of physiological joints is essential for the correct modelling of the physiological kinematic chains under study. However, the complex geometry of articular surfaces and, especially, the important role of ligaments in physiological joint functioning, limits this correspondence. This is the case in some flat joints that can behave like spherical joints such as the shoulder acromioclavicular joint, or of some theoretically two-DOF condyl joints or saddle-shaped ones that can be equated with three-DOF spherical joints if a spinning motion occurs, as will appear for the shoulder sternoclavicular joint. This specificity of each anatomicaljoint complicates the mechanical interpretation but does not exclude it, since it can be considered that each anatomical joint can be ideally represented by one of the classical mechanical pairs. Furthermore, and to a certain extent, the convexity of corresponding articular surfaces can be taken into account in a joint mechanical interpretation: the convex surface being the male part of the joint and the concave surface its female part. This is, however, not so simple in the case of saddleshaped joints and of some ambiguous flat joints. Hence one 

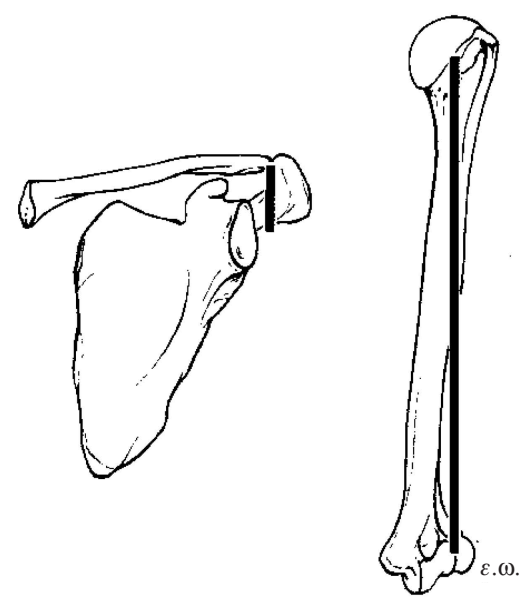

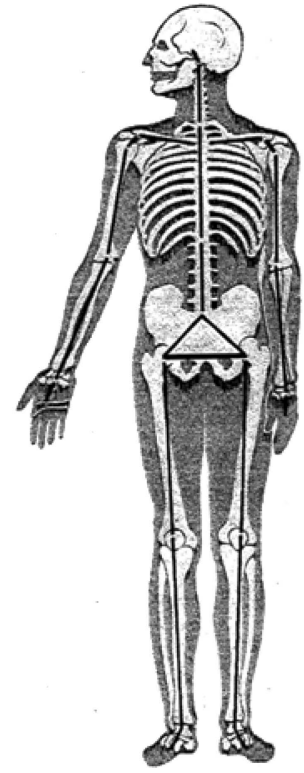

(b)

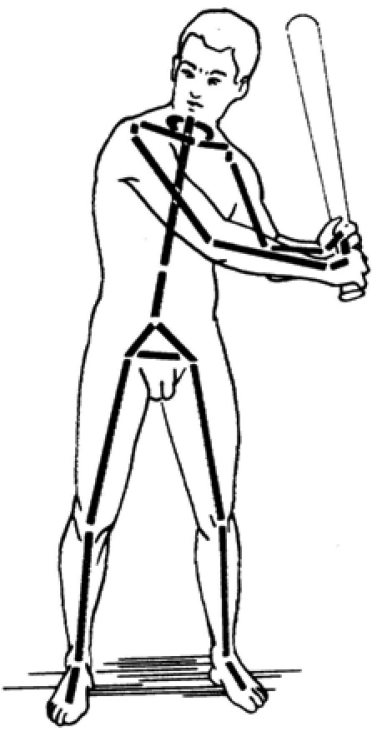

(c)

Figure 1 Dempster's kinematic notation based on a modelling of bones in the form of simple body links: (a) scapula and humerus examples (Dempster 1965); (b and c) application to the skeletal modelling (reprinted from Demspter 1955 for scheme 'b' and from Demspter 1965 for scheme 'c').

reason why the kinematic modelling of the clavicle inside the shoulder girdle is a compound problem.

The modelling of physiological joints cannot be developed independently of a geometric modelling of skeletal bones supporting the joints. In the 1950s, W. Demspter introduced the notion of a body link defined as 'a line of constant length that spans the pin axes' (Dempster 1955, p 560). This simplified representation leads to modelling the skeletal limbs as kinematic chains of rigid links by analogy with the kinematic modelling of machines. More accurately, Dempster models the bones in the form of simple links containing only two joints. For example, he models the humerus as the straight-line segment joining the glenohumeral joint and the elbow joint as illustrated in Figure 1(a). This bone modelling leads to the diagrammatic form of the human skeleton as an arborescence of simple kinematic chains, as illustrated in Figure 1(b) (Demspter 1955) and (c) (Demspter 1965).

Owing to its high synthesis power, Dempster's notation is broadly still used today (see, e.g., Engin and Tümer's extensive paper on shoulder biomechanism (Engin and Tümer 1989; Tümer and Engin 1989). However this method can be criticised for being limited to simple links only, thus ignoring the possibility of links with more than two joints. The scapula is typically a bone containing three joints. Dempster models it as the straight line linking the acromioclavicular joint and the glenohumeral joint, as Figure 1(a) illustrates. By so doing, Dempster ignores the existence of the false scapulothoracic joint, which has to be taken into account in modelling the shoulder girdle. This is the reason why this study suggests, in the spirit of machine and mechanisms theory (Myszka 2002), extending Dempster's body link notation from a simple link-type model, to a complex link-type model. The theory of mechanisms indeed distinguishes 'simple links', which are mechanical links with two joints, from 'complex links,' which are mechanical links having more than two joints. For example, the mechanism in Figure 2(a) is composed of five mobile simple links, four of which are simple links represented in the form of four linear segments, and one of which is a three-joint complex link represented by a hachured triangle, as Figure 2(b) illustrates. In a similar way, we suggest substituting Dempster's linear representation of the scapula by a triangular representation, with the third nodes composed of the centres of the acromioclavicular (AC), glenohumeral (GH) and scapulothoracic (ST) joints, considered as mechanical joints, as illustrated in Figure 2(c).

This triangular kinematic representation of the scapula should not be mistaken for another geometric representation of the scapula, which is sometimes considered, defined by the triangle consisting of the three angles of the scapula (AI [angulus inferior], AS [angulus superior], AA [angulus acromialis]), or the AI with two points TS (trigonum scapulae) and AA (angulus acromialis), as illustrated in Figure 3(a). These purely geometric representations of the scapula have no kinematic meaning in the sense that the nodes of the considered triangles are not associated to the scapula joints. The reproach, of mixing kinematic joint centres with nodes of the scapula geometric representation, can be made of Pronk's model (Pronk 1989), according to which 'the scapula [is modelled] by the beam 


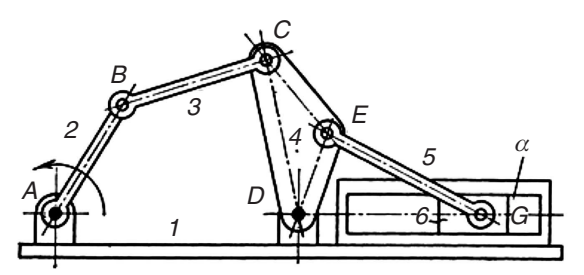

(a)

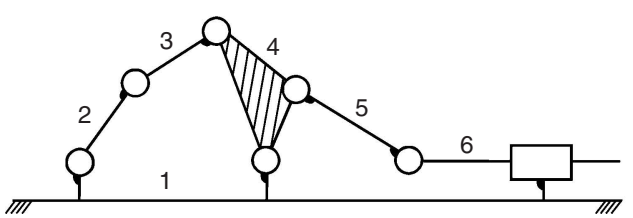

(b)

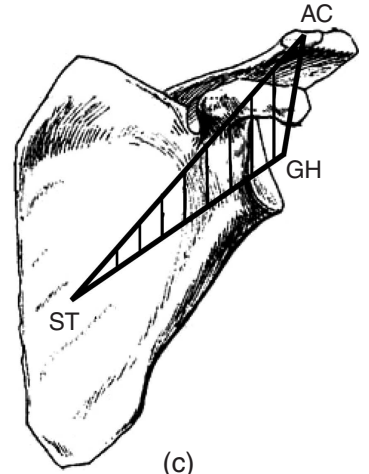

(c)

Figure 2 Proposed extension of Dempster's notation to complex link-type bones: (a) mechanism example with a complex link - the link 4-(from Artobelevski 1977); (b) conventional diagram of the mechanism highlighting the polygonal representation of a complex link; (c) three-joint kinematic representation of the scapula: as in mechanism link 4, the scapula is represented by the polygon joining the three centers, respectively, of the acromioclavicular (AC), glenohumeral (GH) and scapulothoracic (ST) joints.

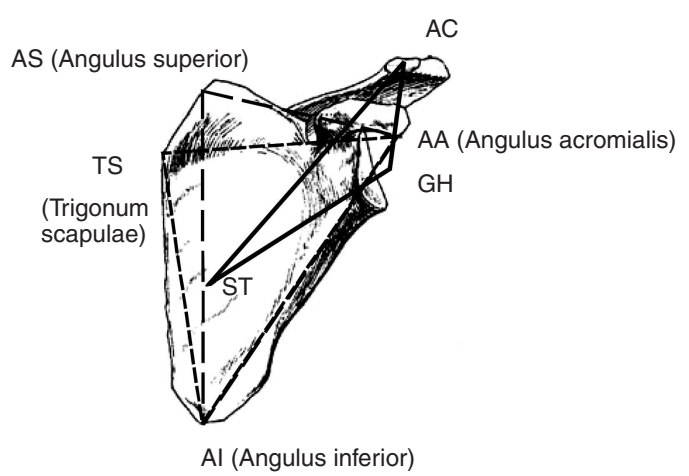

(a)

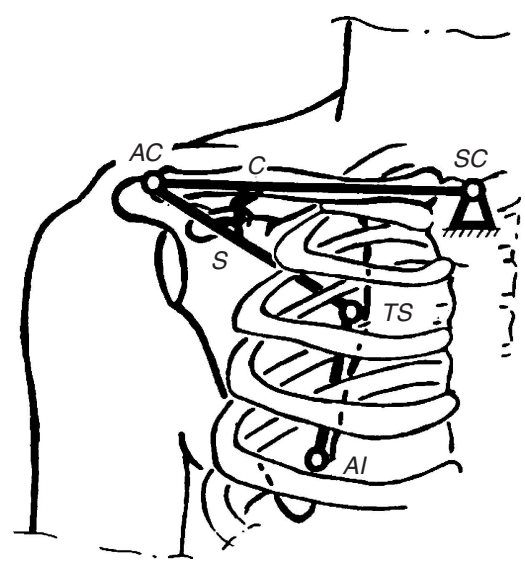

(b)

Figure 3 Triangular representation of the scapula: (a) comparison between the usual representation of the scapula as a triangle joining three remarkable points of its geometry and the proposed one joining the three joints involved in shoulder girdle kinematics; (b) criticism of Pronk's model, mixing 'geometric' and 'kinematic' nodes in the kinematic diagram of the scapula (reproduced from Pronk 1989).

elements AC-TS and TS-AI' (p. 120), as illustrated in Figure 3(b). Although the TS and AI 'points' could be considered as remarkable aspects of the scapular link, helping to putting the link, as sometimes considered in kinematic studies (Myskya 2002), into diagrammatic form, their use in the kinematic definition of the scapular link in the end appears confusing. In particular, Pronk's model of limiting the shoulder girdle to only the scapula and clavicle (Pronk $1989, \mathrm{p} 119)$, is not able to take into account the fundamentally closed-chain nature of the shoulder girdle. This is the reason why we suggest basing our kinematic shoulder complex analysis on the proposed triangular kinematic representation of the scapula. Let it finally be noted that the three nodes considered designate the corresponding joint centres. In the case of spherical-like or universal joints, this is the point around which the joint rotates (although it is well known that the instantaneous centre of rotation is not constant, but is assumed to be). In the case of revolute-like joints, this is the point of the rotation axis whose projection on the gliding surface is in the middle position. And in the case of a planar-like joint, this joint centre corresponds to the middle of the gliding surface. In a more general way, it is important to note that the kinematic notation of complex links can lead to planar representation of the bone as in the case of three-joint bones (case of the scapula) or to non-planar representation in the case of more complex bones such as dorsal vertebrae involving six joints (Kapandji 1974). In any event, owing to the fundamentally spatial nature of physiological joints and in accordance with Demspter's body diagrams, the kinematic representation of bodily mechanisms is fundamentally spatial whether the involving bones have a planar or a spatial representation. 


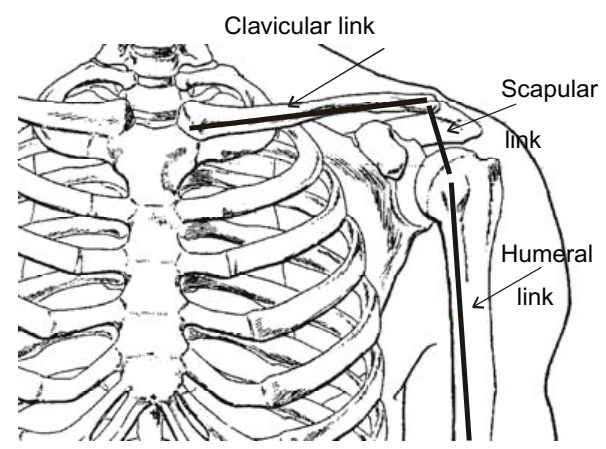

(a)

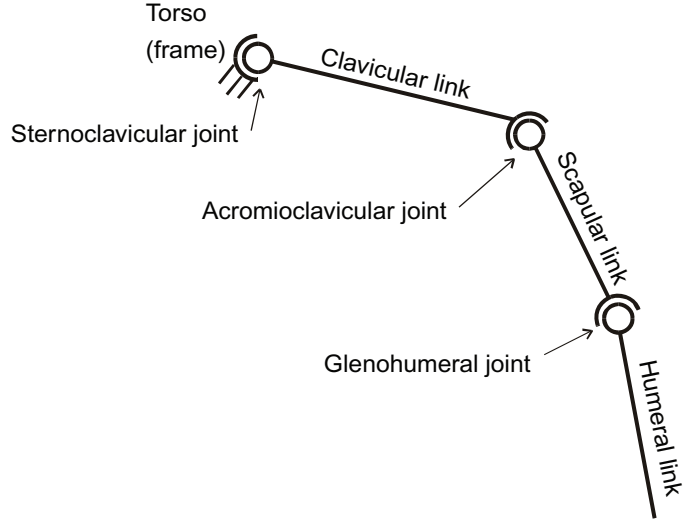

(b)

Figure 4 Kinematic modelling of the shoulder complex as an open kinematic chain: (a) definition of complex shoulder-body links according to Demspter's notation; (b) the corresponding kinematic model of Engin and Tüner.

Furthermore, it is easy to combine this link notation with a joint symbolism not considered by Dempster, but introduced by his successors (Engin and Tümer, for example). Subsequently, the considered Demspter notation will be used in association with the notation of classical mechanical joints as shown in Table 1 , to illustrate our kinematic analysis of the shoulder complex.

\section{THE SHOULDER COMPLEX AS A COMPLEX KINEMATIC CHAIN}

Traditionally, the shoulder complex is defined as a four-main joint system ${ }^{2}$ comprising the sternoclavicular, acromioclavicular, 'false scapulothoracic' and glenohumeral joints. The first three constitute the shoulder girdle while the fourth links the arm to the shoulder. The specificity of the role of the shoulder girdle is to increase the mobility of the humerus, moving in its glenohumeral link. The glenohumeral joint is obviously a ball-and-socket joint that can be modelled by a spherical joint. The modelling of the three joints of the shoulder girdle is more debatable. In particular the modelling of the two clavicular joints is a delicate question. The acromioclavicular or scapuloclavicular is often categorised as a flat joint (Demspter 1965, p 61; Kapandji 1982, p 48; Della Valle et al. 2001, p 321) but is also likened to a ball-and-socket joint (Engin and Tümer 1989; Steindler 1955), which leads to modelling it by a spherical joint. The light convexity of the joint associated to the role of powerful ligaments surrounding it, appears to explain this choice of kinematic

2 Some classical treatises model the shoulder complex with seven joints including the suprahumeral, sternocostal and costovertebral joints (Caillet 1991) but, conversely, the suprahumeral joint "is not an anatomical but a physiological joint" and since "[it] is mechanically linked to the [glenohumeral joint] because any movement in the latter brings about movement in the former" (Kapandji, 1982) it is not necessary to consider it in a global kinematic analysis of the shoulder complex; on the other hand, the sternocostal and costovertebral joints have very low mobility. model. Conversely, the sternoclavicular joint is defined as a saddle-shaped joint that theoretically has two DOFs, but all studies of shoulder physiology underline the existence of a clavicle axial rotation that plays a non-negligible role in upper-extremity elevation, as R. Caillet emphasises in his classical work Shoulder Pain (Caillet 1991, p 42). Subsequently the clavicle axial rotation clearly appears as a DOF. As a consequence, as V. Zatsiorsky emphasises in his recent major publication on the kinematics of human motion, 'kinematically, the sternoclavicular joint functions as a ball-and-socket joint with three DOF' (Zatsiorsky 1998, p 348) in spite of its two-DOF saddle-shaped joint nature.

Thus, the taking into account of all clavicular joint DOFs leads to the modelling of the three true joints of the shoulder complex in the form of three spherical-type joints, as effected in the classical works of Dempster (1965) and Steindler (1955). Accordingly, Tümer and Engin (1989) represented the shoulder complex by an open kinematic chain with three links and three spherical joints, as Figure 4 illustrates.

Such an open-chain modelling, still used today as in, for example, Niemi et al. (1996), is deceptive because it leads to believing that the shoulder complex has nine DOFs. To the best of our knowledge, Dvir and Berne (1978) were the first to suggest a diagrammatic representation of the shoulder complex, which interprets the shoulder girdle as a closed kinematic chain, as Figure 5(a) reproduces. However, no kinematic modelling was deduced by these authors.

This is the interpretation of the false scapulothoracic articulation as a kinematic joint allowing the kinematic modelling of the shoulder girdle. Scapulothoracic articulation gives the shoulder the possibility of gliding over the back of the thorax with possible rotation around a perpendicular axis (the so-called tilting movement). Because the scapula movement is not associated to joint surfaces, the ST articulation is not easily assimilable to a mechanical joint. Van der Helm, in his extensive paper (Van der Helm 1994a,b) considering a shoulder mechanism model derived 

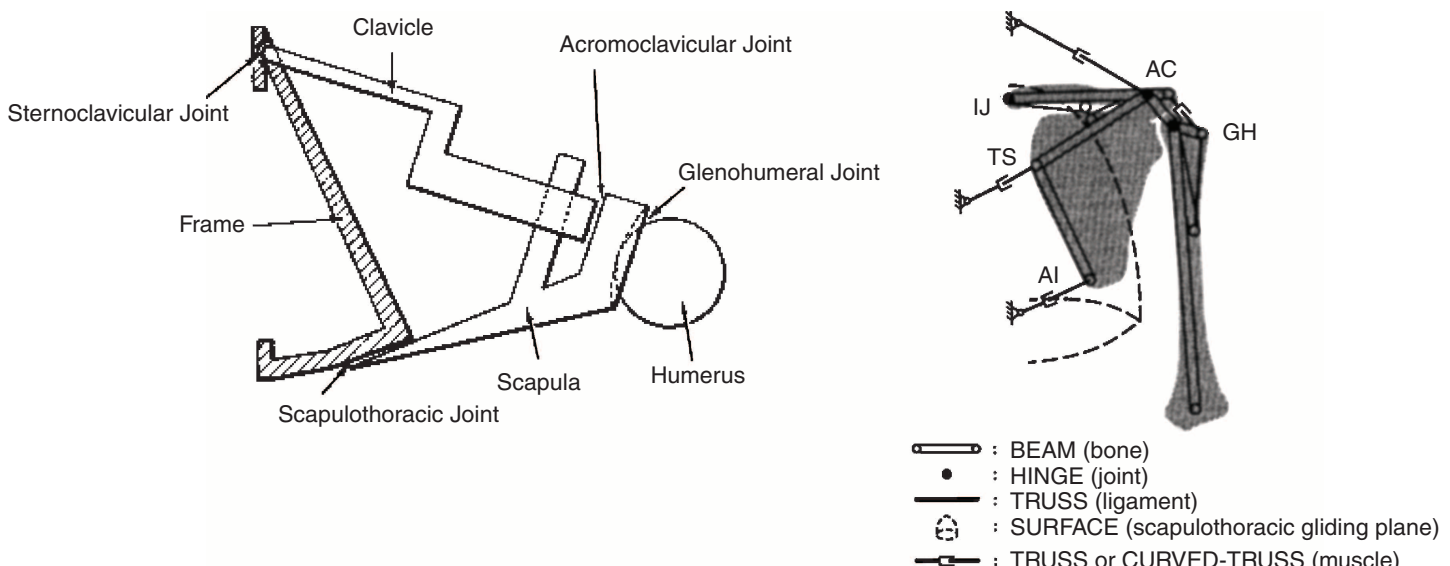

(a)

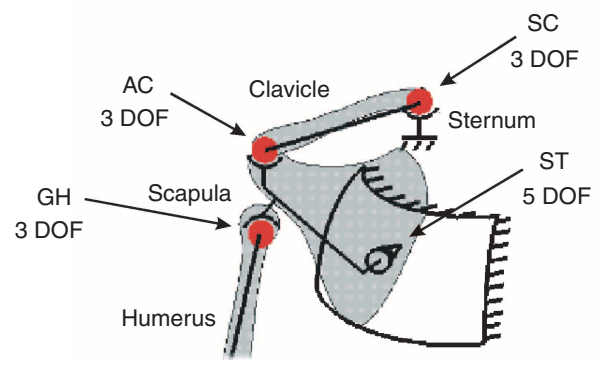

(c) (b)

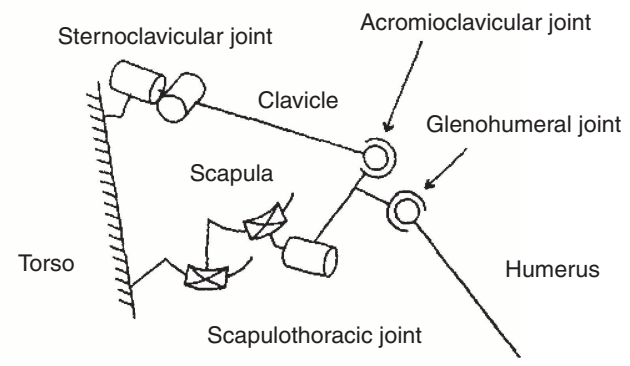

(d)

Figure 5 Some attempts at representing the shoulder-complex mechanism: (a) Dvir and Berne's (1978) scheme; (b) Van der Helm's ( 1994b) diagram; (c) Maurel and Thalmann's (2000) diagram; (d) Lenarcic and Stanisic's (2003) diagram (see comments in the text).

from Pronk's model, defines the shoulder movement as a gliding movement of the two 'beam-bones' AC-TS and TS-AI over the thorax, as Figure 5(b) illustrates. ${ }^{3}$ However, no type of mechanical joint clearly specifies the mobility of these two contact elements. Analysing this modelling problem of the scapula motion over the thorax, W. Maurel suggested interpreting the ST joint as a five-DOF jointpoint on plan, as illustrated in Figure 5(c). This approach certainly facilitates a graphical animation of the upper extremity's natural movements but is difficult to justify from a purely anatomical point of view, because it leads to giving too much freedom to the gliding motion of the scapula over the thorax, which is basically a surface-to-surface contact.

Faced with this difficulty, it is here proposed to model the ST articulation as a simple three-DOF planar joint, whose first two DOFs express the two instantaneous translation motion possibilities of the scapula surface along the thorax surface, the third DOF expressing the rotation motion of the scapula around an axis perpendicular to the plane tangent to the two surfaces in contact. If the thorax, represented by a curved line, is considered as the mechanism

\footnotetext{
3 In a previous paper, Van der Helm considers that "the scapulothoracic gliding plane is modeled by two surface elements which constrain the motions of two points at the medial border of the scapula" (Van der Helm et al. 1991, p 129).
}

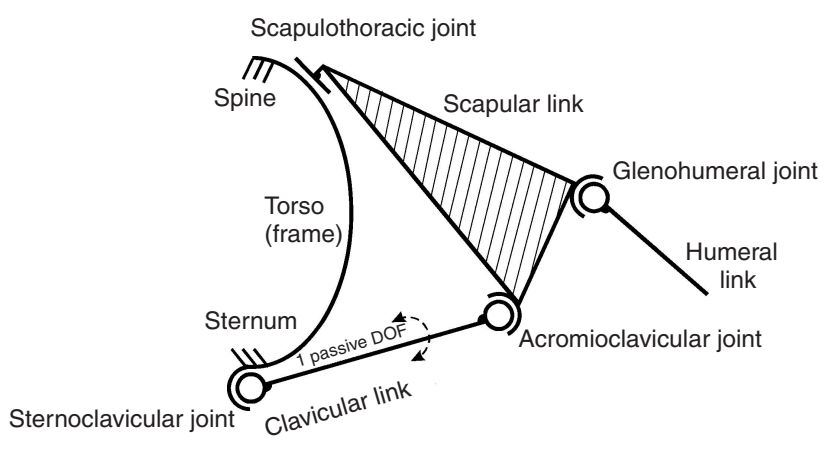

Figure 6 Proposition of a conventional kinematic diagram of the shoulder complex (the left shoulder has been represented to be in accordance with previous Figures 1 to 4 ):

kinematically speaking, the shoulder girdle is composed of three bones (torso, scapular link and clavicular link) jointed respectively by a planar joint and two spherical joints (the passive DOF resulting from the alignment of these two spherical joints is symbolised by a curved double arrow and discussed in the next paragraph); as proposed in Fig. 2(c). the scapula is represented in the form of a hachured triangle whose nodes AC, GH and ST have been replaced by the corresponding conventional joint symbols.

frame, it results in the kinematic diagram of the shoulder complex given in Figure 6 (note that the humerus head and the clavicle ends have been chosen as male parts of 
Table 2 Binary links with passive degrees of freedom*

Pype
Rotation about an axis through the centres of
the ball joints

${ }^{*}$ The text is reprinted from Tsai (1999), but the diagrams have been introduced by the present author.

corresponding spherical joints). This diagram clarifies Tümer and Engin's by closing the shoulder girdle kinematic chain and Dvir and Berne's by introducing the corresponding joint models. In comparison with the pseudoconventional Pronk and Maurel diagrams it tries to highlight a more formal representation of the bio-mechanism, independent of any simplified anatomical diagram on which it would be drawn, and in comparison with the recent Lenarcic and Stanisic (2003) formal diagram of Figure 5(d), it aims to show the interest of using a correspondence table between physiological joints and mechanical joints.

\section{ESTIMATE OF THE MOBILITY OF THE SHOULDER COMPLEX}

Mobility $F$ of a spatial kinematic chain of $n$ links one of which is the fixed frame, and which are jointed by $j$ articulations is, in general, defined as the difference between the mobility without any constraint of the $(n-1)$ mobile links and the total number of constraints imposed by each joint, i.e.:

$$
F=6(n-1)-\sum_{i=1}^{j} c_{i} .
$$

Since the number $c_{i}$ of constraints imposed by a joint is equal to the difference between the six DOFs of space and the DOF permitted by the joint, we obtain

$$
\begin{aligned}
F & =6(n-1)-\sum_{i=1}^{j}\left(6-f_{i}\right) \\
& \Rightarrow F=6(n-1-j)+\sum_{i=1}^{j} f_{i} .
\end{aligned}
$$

This equation is known as the Grübler-Kutzbach formula. In the case, for example, of the serial chain composed of the shoulder girdle and of the humeral link - that is, the thorax being considered as the fixed frame of a three-link chain with two joints-its application leads to

$$
\begin{aligned}
F_{\text {shoulder complex }} & =6(3-1-2)+F_{\text {shoulder girdle }}+3 \\
& \Rightarrow F_{\text {shoulder complex }}=F_{\text {shoulder girdle }}+3 .
\end{aligned}
$$

which corresponds to the intuitive meaning. Conversely, as Lenarcic and Stanisic clearly show (Lenarcic 2003), the Grübler-Kutzbach formula very relevantly applies to the estimate of the number of degrees of freedom of the shoulder girdle, which is too often determined in a purely intuitive way. It is, however, important to note that Grübler-Kutzbach is not universal and can create difficulty in certain geometric situations (e.g., the so-called overconstrained mechanisms that require special links to achieve mobility). Expecting these situations, it is possible, as Tsai emphasises (Tsai 1999), to extend the Grübler-Kutzbach criterion in taking into account the possible presence of passive DOF. Besides obvious joint redundancies, Tsai distinguishes three types of binary links with passive DOFs (Table 2).

If we note $f_{\mathrm{p}}$ as the number of passive DOF, Tsai puts forward the extended Grübler-Kutzbach formula, such that

$$
F=6(n-1-j)+\sum_{i=1}^{j} f_{i}-f_{\mathrm{p}} .
$$

This is the formula which we suggest should apply to the calculation of shoulder girdle mobility. Under the assumptions made in a previous paragraph, $n$, the number of the mechanism links, is equal to 3 (torso, clavicular link, 
scapular link), $j$, the number of joints, is equal to 3 (two spherical joints and one planar joint) and all $f_{i}$ are equal to 3. But it is our opinion that the axial clavicular rotation must be considered as a passive DOF due to the fact that it corresponds to a rotation around an axis passing through the centre of two spherical joints, as illustrated in the S-S diagram of Table 2. This interpretation corresponds to the non-motor role of this joint motion: in point of fact, according to Inman et al. (1944) the axial clavicular rotation plays an anatomical role essential for arm elevation, because it helps draw out the ligaments enclosing the acromioclavicular joint. Thus, within the framework of a kinematic model that does not take into account any ligamentary movements, it appears as one passive DOF Taking this passive DOF into account, the application of the GrüblerKutzbach formula leads to the following:

$$
F_{\text {shoulder girdle }}=6(3-1-3)+3 \times 3-1=2 \mathrm{DOF}
$$

Note that we finally obtain the same end result as that given by Lenarcic and Stanisic 2003), but the present approach appears more efficient in that it emphasises the specific role of the clavicle within the shoulder girdle. Consequently, the total shoulder-mobility complex is estimated to be 5 .

Beyond this numerical result, we wish to emphasise the generality of our approach that can be adapted to other assumptions concerning the mobility of the links of the shoulder complex; for example, if we adopt the assumption of Maurel and Thalmann's model (Fig. 5(c)) giving five DOFs to the scapulothoracic joint-with the same assumptions as ours concerning the other joints-the shoulder girdle would have a mobility of $6(3-1-3)+3 \times 2+5-1=$ $4 \mathrm{DOF}$. It is interesting to note that the final mobility result can serve as a feed-back of the mechanism's separate joint mobility assumptions; in the case of Maurel and Thalmann's model a 4 high global shoulder girdle mobility appears high physiologically speaking and it can be asked if among four corresponding generalised coordinates some of them have no negligible variations. Our proposed two-DOF shoulder girdle model is obviously a simplified one, but it notably aims to emphasise major corresponding generalised coordinates from the knowledge of which, in particular, as will be seen later, it is possible to derive an equivalent more suitable kinematic model for further kinematic and dynamic simulations.

Furthermore, in accordance with the principles of the mechanism theory our approach clearly distinguishes the joints, which are concerned by the mobility analysis from the actuators (the muscles) and their annexes (the ligaments), which are not so concerned. In this way, our approach differs from the one developed by Van der Helm, for example, which mixes purely kinematic constraints with muscular or ligamentary constraints. Generally speaking, a ligament cannot reduce the mobility efficiency of a joint, but can only limit its range of motion: for example, the 'conoid ligament', considered in his model as a constraint in Van der Helm's model—and clearly shown in Figure 3(b) of Pronk's model, from which it derives - (Van der Helm 1994a,b), plays the part, according to Kapandji, of a limitation of the angle between the clavicle and the scapula but does not determine joint mobility (Kapandji 1982, p. 52). Moreover, in the absence of a general formula for determining the mobility of a complex kinematic chain, the mobility the authors confer on the set 'shoulder-complex/ elbow flexion-extension' is very questionable: The sternoclavicular, acromioclavicular, glenohumeral and elbow joint yield $10 \mathrm{DOF}$, the conoid ligament and the scapulothoracic gliding plane impose 3 kinematic constraints which yields a model with 7 kinematic DOF. (Happee and Van der Helm 1995, p 1181)

Without wishing to criticise the important work of an author of the stature of Van der Helm and his team on shoulder biomechanics (brilliantly synthesised in a recent paper, Gupta and Van der Helm 2004, discussing Lewin's (1997) audacious assumption) of a tensegrity structure of the scapula) our remark aims at stressing the interest of a rigorous and purely kinematic analysis of any physiological mechanism in helping further dynamic analysis of it.

\section{DETERMINATION OF AN EQUIVALENT KINEMATIC MODEL OF THE SHOULDER COMPLEX}

The determination of shoulder girdle mobility raises the question of putting the emphasis on an equivalent kinematic model in the form of an open chain, with the same mobility. Such an equivalent model could be useful in certain problems or applications, which do not need to mimic the whole of the skeletal structure of the shoulder girdle. The search for a rigorously equivalent model is a particularly difficult problem. We suggest a simplified approach, which aims at highlighting an 'equivalent clavicle'. Let $S$ be the centre of the sternoclavicular joint to which a fixed reference frame can be associated. Let $G$ be the centre of the glenohumeral joint. Since the shoulder girdle has a twoDOF mobility, it is clear that two independent coordinates define the position of $G$ in space. Two joint variables $\theta_{1}$ and $\theta_{2}$ modelling the mobility of the sternoclavicular joint can be chosen as independent generalised coordinates. Because the distance between the stenoclavicular joint and the glenohumeral joint is clearly not constant, a third, linear and dependent, parameter has to be considered to express variable length $S G$. An equivalent model results composed of two aligned links, the first one being joined to the sternum by a universal joint of variables $\theta_{1}$ and $\theta_{2}$, the second joined to the first by a prismatic joint dependent on $\theta_{1}$ and $\theta_{2}$, as illustrated in Figure 7. From a biomechanical point of view, this model very imperfectly expresses the orientation of the glenohumeral socket. However, it seems to us relevant because it expresses the fundamental kinematic role of the shoulder girdle: to position the glenohumeral joint in space. The suggested model differs from the more recent Lenarcic and Stanisic (2003) model, which considers 


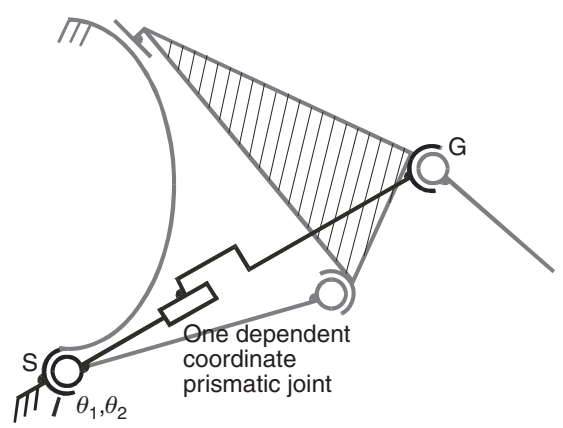

Figure 7 Equivalent kinematic model of the shoulder girdle in the form of an 'equivalent clavicle'.

the rotation centre of the scapula as an intermediate element; however, this more complete scapula-centred model seems to us difficult to prove in a rigorous manner owing to shoulder movement complexity, and more complex to put into operation than our one.

It is also important to note that such a model with a passive DOF - the DOF of the joint dependent on the universal joint motion - appears inadequate for use in practice. This is why we could like to emphasise the interest of a shoulder girdle model reduced to a simple link - that is, an equivalent clavicle of constant length - joined to the thorax by a universal joint. Associated to a traditional 7R anthropomorphic robot model, a serial architecture of the human arm results in four links: clavicle, arm, forearm, handand nine joints whose definition can be put in correspondence with joint physiology, as Figure 8 illustrates, where the $\theta_{1}$ and $\theta_{2}$ joint variables of the equivalent kinematic model of the shoulder girdle are interpreted as clavicular flexion-extension and clavicular abduction-adduction angles.

This structure is not new. It has already been considered in some earlier robotics (Jacobsen 1973) or biomechanics studies (Lenarcic and Umek 1994). It is, for example, used in the 103-DOF SANTOS digital human modelling environment of the Iowa University Virtual Soldier Research programme (Beck et al. 2004). The rigorous demonstration of shoulder girdle mobility confirms its value. Furthermore, it is useful to recall the importance given to the clavicle by E. A. Codman (1934) in his fundamental essay on the shoulder:

We are proud that our brains are more developed than those of animals; we might also boast of our clavicles. It seems to me that the clavicle is one of man's greatest skeletal inheritances, for he depends to a great extent than most animals except the apes and monkeys, on the use of his hands and arms. The clavicle holds the shoulder away from the body and therefore permits us to use our arms with power and skill in abduction, and adduction to a degree which few animals except the monkeys can approach (pp 7-8).

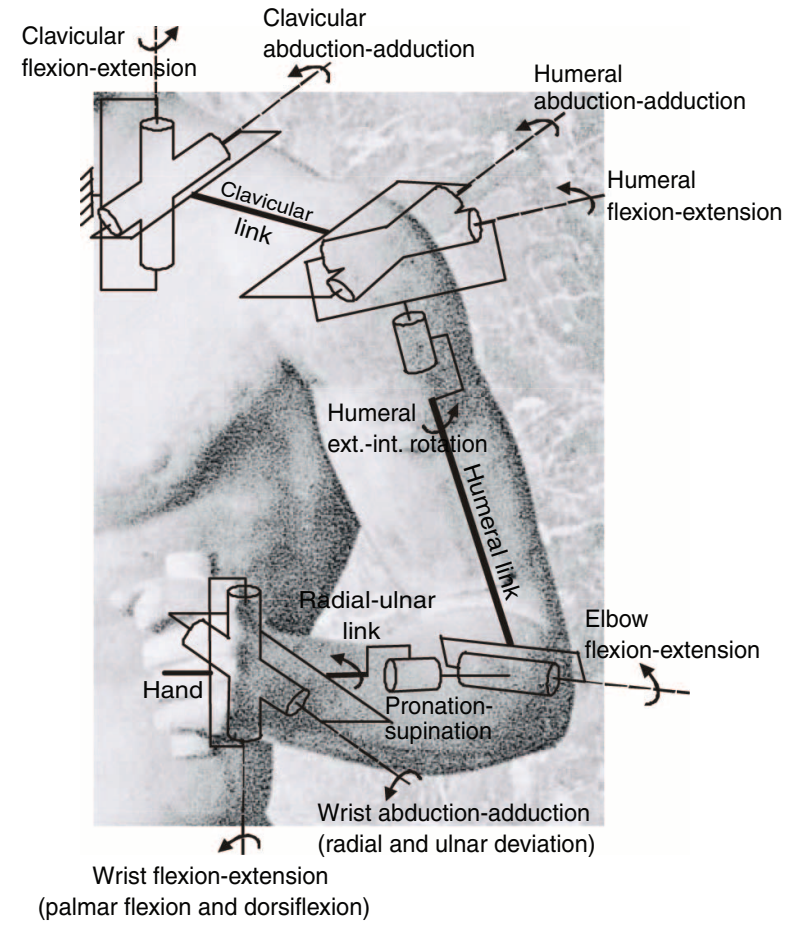

Figure 8 Kinematic model of the human upper extremity as an open kinematic chain with four links (clavicle or clavicular link, arm or humeral link, forearm or radial-ulnar link, hand) and nine DOFs: the shoulder girdle appears in the form of the 'equivalent clavicle' (denoted clavicular link) jointed to the torso by the two-DOF clavicular flexion-extension and clavicular abduction-adduction.

\section{CONCLUSION}

We have tried in the framework of this article to throw light on the contribution of mechanism theory to the difficult problem of kinematic modelling of the shoulder complex.

The notion of a 'complex link'-in the meaning given by mechanism theory-seemed relevant to us so as to widen Dempster's historical notation to bones with multiple joints such as the scapula.

At the same time, the Grübler-Kutzbach formula can be advantageously applied to biomechanisms, provided that an agreement is reached on the mechanical interpretation of the physiological joints concerned in the skeletal subsystems under study. The application of this mechanismtype approach to the shoulder complex has led us to demonstrate that shoulder girdle mobility is equivalent to two, and that of the shoulder complex to five. The simplified model we have considered does not claim to be a substitute for more complex models of the 'shoulder rhythm' as theorised and developed by Hogfors et al. (1991). But using it validates a classical kinematic model of the upper limb with four links - clavicular, arm, forearm and hand-and nine DOFs useful for biomechanical studies that have no need to take into account the muscles actuating shoulder-complex bones, but also in computer animation depicting humanoid robotics. 


\section{ACKNOWLEDGMENT}

We acknowledge the anonymous referees for their help through their very pertinent remarks and proposals.

\section{REFERENCES}

Abdel-Malek K, Yank J, Brand R, Tanbour E. 2004. Towards understanding the workspace of human limbs. Ergonomics, 47-13:1386-405.

Artobolevski I. 1977. Théorie des mécanismes et des machines. Editions MIR-Moscou (translated from the Russian).

Beck S, Abdel-Malek K, Arora J. 2004. Virtual Soldier Research Program-The University of Iowa. Web site: www.digital-humans.org.

Biryukova EV, Roby-Brami A, Frolov AA, Mokthari M. 2000. Kinematics of human arm reconstructed from spatial tracking system recordings. 7 Biomech, 33: 985-95.

Caillet R. 1991. Shoulder Pain, 3rd ed.. Philadelphia: F.A. Davis.

CHARM Esprit Project: Comprehensive Human Animation Resource Model. Esprit Project 9036. URL: http://ligwww.epfl.ch/ maurel/CHARM.

Codman EA. 1934. The Shoulder. (Malabar, FL: Robert E. Kreiger Publishing Co (Originally published : T. Todd Co.).

Dayanidhi S, Orlin M, Kozin S, Duff S, Karduna A. 2005. Scapular kinematics during humeral elevation in adults and children. Clin Biomech, 20:600-6.

De Groot, Brand R. 2001. A three-dimensional regression model of the shoulder rhythm. Clin Biomech, 16:735-43.

Della Valle CJ, Rokito AS, Birdzell MG, Zuckerman JD. 2001. Biomechanics of the shoulder. In Nordin M and Frankel VH, eds. Basic Biomechanics of the Musculoskeletal System, 3rd ed. Philadelphia: Lippincott Williams \& Wilkins, chap. 12, p. 318-39.

Dempster WT. 1955. The Anthropometry of body action. Ann N Y Acad Sci, 63:559-85.

Dempster WT. 1965. Mechanisms of shoulder movement. Arch Phys Med Rehabil, January:49-70.

Dvir Z, Berne N. 1978. The Shoulder complex in elevation of the arm: A mechanism approach. $\mathcal{F}$ Biomech, 11:219-25.

Engin AE, Tümer ST. 1989. Three-dimensional kinematic modelling of the human shoulder complex-Part 1: Physical model and determination of joint sinus cones. 7 Biomech Eng, 111:107-12.

Gupta S, Van der Helm FCT. 2004. Load transfer across the scapula during humeral abduction. 7 Biomech, 37:1001-9.

Happee R, Van der Helm FCT. 1995. The control of shoulder muscles during goal directed movements, an inverse dynamic analysis. $\mathcal{F}$ Biomech, 28(10):1179-91.

Hogfors C, Peterson B, Sigholm G, Herberts P, 1991. Biomechanical model of the human shoulder joint-II. The shoulder rhythm. 7 Biomech, 24(8):699-709.

Holzbaur KRS, Murray WM, Delp SL. 2005. A model of the upper extremity for simulating musculoskeletal surgery and analysing neuromuscular control. Ann Biomed Eng, 33(6):829-40.

Inman VT, Saunders JB, Abbott LC. 1944. Observation on the function of the shoulder joint. 7 Bone Foint Surg, 26A:1-30.

Jacobsen SC. 1973. Control systems for artificial arms. IEEE Conference on Systems, Man and Cybernetics. Detroit: IEEE Press, p. 298-303.
Kapandji IA. 1982. Physiology of the Joints, Vol. 1, Upper Limb, 5th ed. Oxford, UK: Churchill Livingston.

Kapandji IA. 1974. Physiology of the Joints, Vol. 3, Trunk and Vertebral Column, 2nd ed. Oxford, UK: Churchill Livingston.

Karduna AR, McClure PW, Michener LA. 2000. Scapular kinematics: Effects of altering the Euler angle sequence of rotations. 7 Biomech, 33:1063-8.

Lenarcic J, Stanisic M. 2003. A humanoid shoulder complex and the humeral pointing kinematics. IEEE Trans Robotics Automat, 19(3): 499-506. (Please note that the figures of the original paper were mixed up and that their placing was corrected in an addendum published in the August 2003 edition of the journal).

Lenarcic J, Umek A. 1994. Simple Model of Human Arm Reachable Workspace. IEEE Tran Syst Man Cybernet, 24(8):1239-46.

Lewin SM. 1997. Putting the shoulder to the wheel: A new biomechanical model for the shoulder girdle. Biomech $S_{c i}$ Instrument, 33:412-7.

Magermans DJ, Chadwick EK, Veeger HEJ, Van der Helm FCT. 2005. Requirements for upper extremity motions during activities of daily living. 7 Biomech, 20:591-9.

Maurel W, Thalmann D. 1999. A case study on human upper limb modelling for dynamic simulation. Comput Methods Biomech Biomech Eng, 1(2):1-17.

Maurel W, Thalmann D. 2000. Human shoulder modelling including scapulo-thoracic constraint and joint sinus cones. Comput Graphics, 24:203-18.

McClure P, Michener LA, Sennett BJ, Karduna AR. 2001. Direct 3-dimensional measurement of scapulae kinematics during dynamic movements in vivo. 7 Shoulder Elbow Surg, 10(3):269-77.

Myszka DH. 2002. Machines \& Mechanisms, 2nd ed. Upper Saddle River, NJ, USA: Prentice Hall.

Niemi J, Nieminen H, Takala E-P, Viikari-Juntura E. 1996. A static shoulder model based on a time-dependent criterion for load sharing between synergistic muscles. 7 Biomech, 29(4):451-60.

Prokopenko RA, Frolov AA, Biryukova EV, Roby-Brami A. 2001. Assessment of the accuracy of a human arm model with seven degrees of freedom. $\mathcal{F}$ Biomech, 34:177-85.

Pronk GM. 1989. A kinematic model of the shoulder girdle: A résumé. 7 Med Eng Technol, 13(1-2):119-23.

Rab G, Petuskey K, Bagley A. 2002. A method for determination of upper extremity kinematics. Gait Posture, 15:113-9.

Raikova R. 1992. A general approach for modelling and mathematical investigation of the human upper limb. $\mathcal{F}$ Biomech, 25(8):857-67.

Scepi M, Faure JP, Ridoux N, Kamina P, Richer J-P. 2004. A three-dimensional model of the shoulder girdle. Forces developed in deltoid and supraspinatus muscles during abduction. Surg Radiol Anat, 26(4):290-96.

Steindler A. 1955. Mechanics of Shoulder-Arm Complex, in Kinesiology of the Human Body under Normal and Pathological Conditions. Springfield: Charles C. Thomas Publisher.

Tsai L-W. 1999. Robot Analysis-The Mechanics of Serial and Parallel Manipulators. New York: John Wiley \& Sons.

Tümer ST, Engin AE. 1989. Three-dimensional kinematic modelling of the human shoulder complex-Part 2 : Mathematical modelling and solution via optimization. $\mathcal{F}$ Biomech Eng, 111:113-21. 
Van der Helm FCT. 1994a. Analysis of the kinematic and dynamic behaviour of the shoulder mechanism. 7 Biomech, 27(5):527-50.

Van der Helm FCT. 1994b. A finite element musculoskeletal model of the shoulder mechanism. $\mathcal{F}$ Biomech, 27(5):55169.
Van der Helm FCT, Veeger HEJ, Pronk GM, Van der Woude LHV, Rozendal RH. 1991. Geometry parameters for musculoskeletal modelling of the shoulder system. F Biomech, 25(2):129-44.

Zatsiorsky VM. 1998. Kinematics of Human Motion. Human Kinetics. Champaign, IL, USA. 

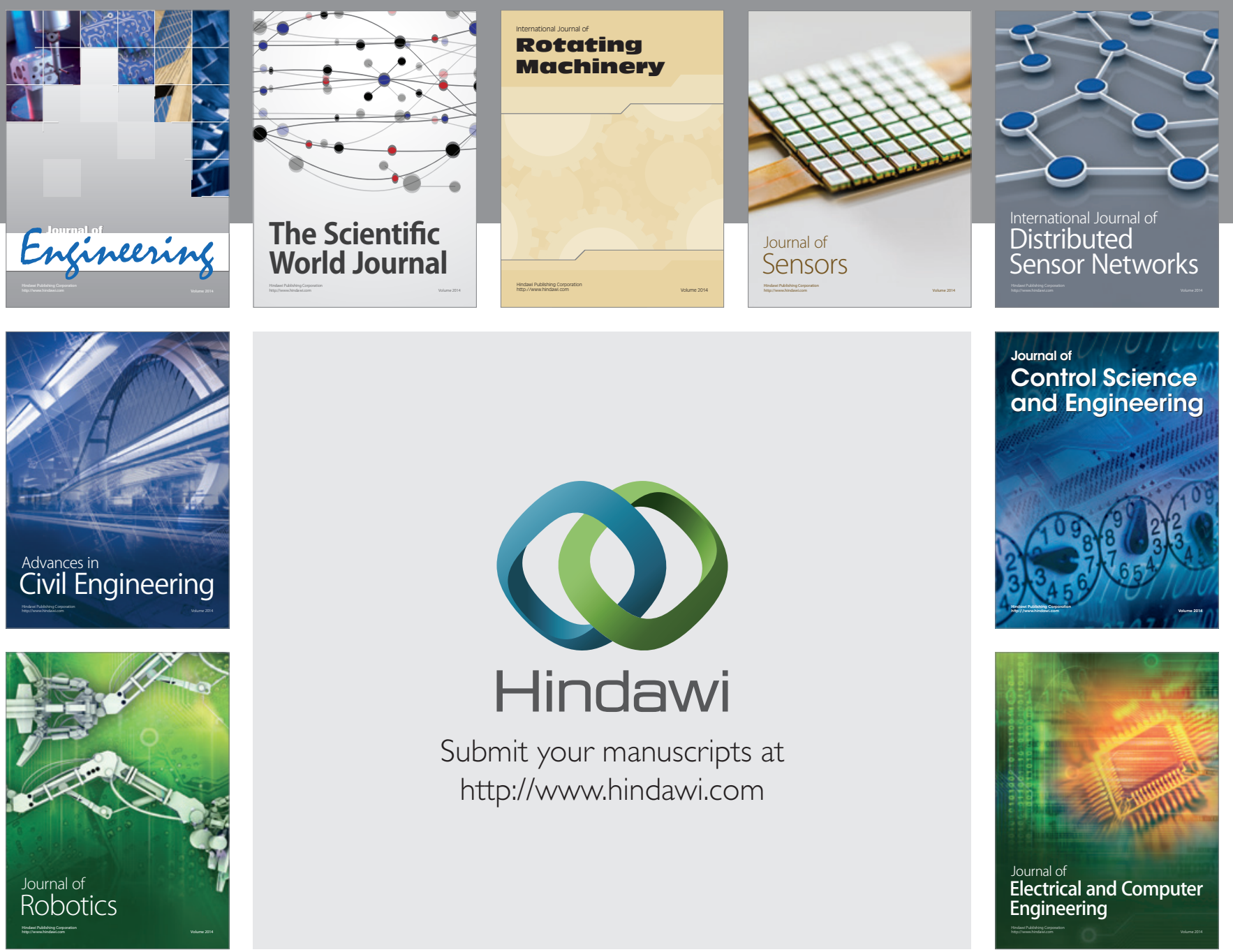

Submit your manuscripts at

http://www.hindawi.com
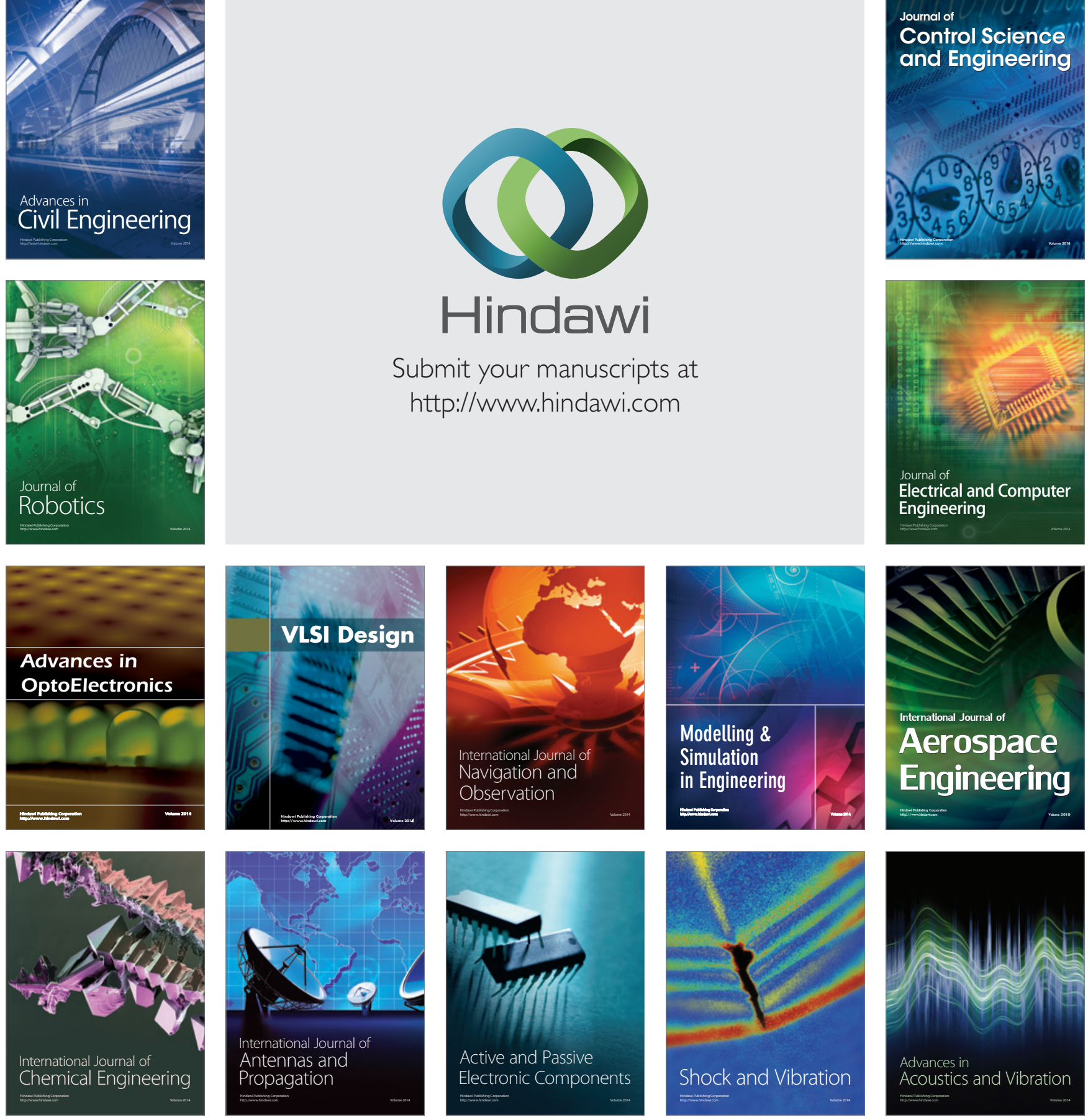